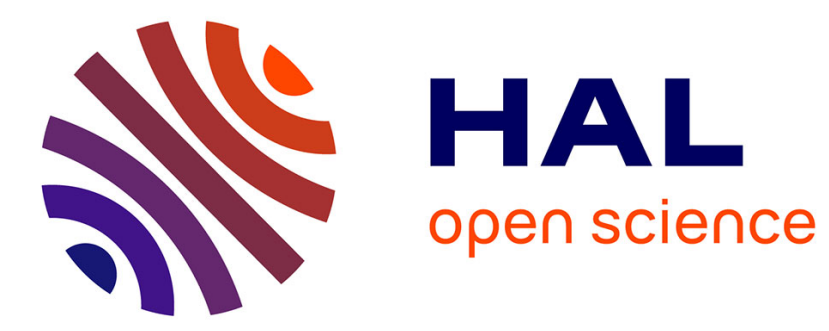

\title{
Unknown input observers for switched nonlinear discrete time descriptor systems
}

Damien Koenig, Benoît Marx, Denis Jacquet

\section{To cite this version:}

Damien Koenig, Benoît Marx, Denis Jacquet. Unknown input observers for switched nonlinear discrete time descriptor systems. IEEE Transactions on Automatic Control, 2008, 53 (1), pp.373-379. 10.1109/TAC.2007.914226 . hal-00193514

\section{HAL Id: hal-00193514 https://hal.science/hal-00193514}

Submitted on 25 Nov 2021

HAL is a multi-disciplinary open access archive for the deposit and dissemination of scientific research documents, whether they are published or not. The documents may come from teaching and research institutions in France or abroad, or from public or private research centers.
L'archive ouverte pluridisciplinaire HAL, est destinée au dépôt et à la diffusion de documents scientifiques de niveau recherche, publiés ou non, émanant des établissements d'enseignement et de recherche français ou étrangers, des laboratoires publics ou privés. 


\section{Unknown input observers for switched nonlinear discrete time descriptor systems}

\author{
D. Koenig, B. Marx and D. Jacquet
}

\begin{abstract}
In this paper, a linear matrix inequality (LMI) technique for state estimation of discrete-time, nonlinear switched descriptor systems is developed. The considered systems are composed of linear and nonlinear parts. An observer giving a perfect unknown input (UI) decoupled state estimation is proposed. Sufficient conditions of global convergence of observers are proposed. Numerical examples are given to illustrate this method.
\end{abstract}

Index Terms-Switched descriptor systems, hybrid systems, unknown input observers, poly-quadratic stability.

\section{INTRODUCTION}

Switched control and/or observer systems has recently received much attention. Switched systems belong to a special class of hybrid systems. They are defined by a collection of dynamical (linear and/or nonlinear) subsystems together with a switching rule that specifies the switching between these subsystems. A survey on basic problems in switched system stability and design is available in [26] (see the references therein). Many such problems occur in practice: Power converter systems where the switching signal is determined by pulse with PWM modulation and gain scheduling control systems are examples among many others. One can study the existence of a switching rule that ensures stability of the switched system. One can assume that the switching sequence is not known a priori and look for stability results under arbitrary switching sequences. On the one hand, most of the contributions in this field deal with stability analysis and control synthesis [7], [18]. On the other hand, unknown input observers (UIO) have been widely studied for nonsingular systems [9], [29], singular system [10], [6], [16], nonlinear descriptor systems [17] and recently for switched nonsingular systems [20]. Nevertheless, there is no result extending the method mentioned in [20] to the general representation of switched nonlinear descriptor systems although many practical systems can be described by them [2] and their fault diagnosis may be based on UIO design [21].

As mentioned in [32], there are generally two broad approaches for nonlinear observer design. In the first approach, the objective is to find a coordinate transformation so that the state estimation error dynamics are linear in the new coordinates and them linear techniques can be performed [13], [14], [30]. Necessary and sufficient conditions have been established [30], [19] for the existence of such a coordinate transformation. The second approach does not need the transformation and the observer design is directly based on the original system. Because of the complexity of nonlinear systems, a

D. Koenig and D. Jacquet are with the Gipsa-lab (UMR 5216, CNRSINPG-UJF), Institut National Polytechnique de Grenoble, BP 46, 38402 Saint Martin d'Hères, Cedex, France (e-mail : Damien.Koenig@inpg.fr).

B. Marx is with the Centre de Recherche en Automatique de Nancy (CRAN - UMR 7039), Nancy-Université, CNRS, 2 avenue de la forêt de Haye, 54516 Vandoeuvre-les-Nancy Cedex, France. lot of directly designing methods have been developed. For instance, Praly et al. [22], [15], [28] contributed some results on observer design using high-gain techniques. Besancon and Hammouri [3] and Dawson [12], studied the observer design from the solution of Riccati equation for Lipschitz nonlinear systems. Adaptive observers have been proposed for special classes of nonlinear systems [23], [5]. For the class of global Lipschitz nonlinear systems, existence condition have been established for full-order observer and also for reduced-order observers respectively in [24] and [32]. The design method is based on the solution of a Riccati equation. More recently, based on the linear matrix inequality (LMI) approach both proportional and proportional integral observer for nonlinear descriptor system has been proposed in [17]. According to remark 1 in [17] the nonlinear systems considered in this paper is more general than [5], [24], [32]. Moreover, we proposed to extend the design of a proportional observer for an unsquare (rectangular) switched descriptor system which include both UI and Lipschitz nonlinearities. The systems considered are also in a general form and seem to be the first using convex optimization. Briefly an extension of UIO design for linear system to nonlinear system is proposed.

This note is organized as follows. Section II presents the problem statement. A design method of the proportional observer and the main results of this note are given in section III. In section IV the performance of the proportional switched observer is evaluated through two numerical examples. The proof of the detectability condition is provided in appendix. Finally, section V concludes the paper.

\section{PROBLEM FORMULATION}

Consider the switched nonlinear descriptor systems

$$
\begin{aligned}
& E_{\alpha(k+1)} x_{k+1}=A_{\alpha(k)} x_{k}+F_{\alpha(k)} d_{k}+H_{\alpha(k)} \phi_{k} \\
& y_{k}=C_{\alpha(k)} x_{k}+G_{\alpha(k)} d_{k}
\end{aligned}
$$

where $E_{\alpha(k+1)}, A_{\alpha(k)} \in \mathbb{R}^{p \times n}$ are in the general form and may be rectangular, $F_{\alpha(k)} \in \mathbb{R}^{p \times q}, H_{\alpha(k)} \in \mathbb{R}^{p \times n_{\phi}}$, $C_{\alpha(k)} \in \mathbb{R}^{m \times n}, G_{\alpha(k)} \in \mathbb{R}^{m \times q}, p \leq n, x \in \mathbb{R}^{n}, d \in \mathbb{R}^{q}$, $\phi_{k}=\phi\left(x_{k}, u_{k}, k\right): \mathbb{R}^{n} \times \mathbb{R}^{n_{u}} \times \mathbb{N} \rightarrow R^{n_{\phi}}$ and $y \in \mathbb{R}^{m}$ denote respectively the descriptor vector, the unknown input vector, the nonlinearity vector and the output vector. In the sequel, disturbances or partial inputs which are inaccessible are called UI. The signal $u \in \mathbb{R}^{n_{u}}$ is the control input vector. $\alpha(k)$ is a piecewise constant switching signal taking value from the finite index set $\varepsilon=\{1,2, \ldots, h\}$. At a switching time $k$, we have $\alpha(k-1) \neq \alpha(k)$. The ordered sequence of the switching times is said to be the switching time sequence of the switching signal. It is assumed that the switching time sequence is real-time accessible, depending on the control input or on the measured output, or using a finite automation or any strategy. $\left\{\left(E_{i}, A_{i}, F_{i}, H_{i}, C_{i}, G_{i}\right): i \in \varepsilon\right\}$ are a family of matrices parameterized by an index set $\varepsilon=\{1,2, \ldots, h\}$ and $i=\alpha(k)$. Moreover, $\alpha(k)=i$ and $\alpha(k+1)=j$ means that the matrices $\left(E_{j}, A_{i}, F_{i}, H_{i}, C_{i}, G_{i}, D_{i}\right)$ are activated.

Notation 1: $(.)^{T}$ stands for the transpose matrix and $(*)$ is used for the blocks induced by symmetry, (.) $>0$ denotes a symmetric positive definite matrix, $(.)^{+}$is the pseudoinverse 
matrix, $(.)^{\perp}$ is the orthogonal complement, $\|$.$\| stands for the$ Euclidean norm, $(.)_{k_{+}}$stands for $(.)_{\alpha(k), \alpha(k+1)}$, for instance $T_{k_{+}}=T_{\alpha(k), \alpha(k+1)}$.

Remark 1: The orthogonal complement $A^{\perp}$ for a real $n \times p$ matrix $A$ with rank $q$ is defined as $(n-q) \times n$ matrix such that $A A^{\perp}=0$ and $A^{\perp} A^{\perp T}>0$.

Assumptions: In the sequel it is assumed that

A1 the nonlinearity $\phi\left(x_{k}, u_{k}, k\right)$ is globally Lipschitz in $x$ with Lipschitz constant $\gamma$, i.e.,

$$
\begin{aligned}
\left\|\phi\left(x_{k}, u_{k}, k\right)-\phi\left(\hat{x}_{k}, u_{k}, k\right)\right\| & \leq \gamma\left\|x_{k}-\hat{x}_{k}\right\| \\
\forall u & \in \mathbb{R}^{n_{u}}, k \in \mathbb{N}
\end{aligned}
$$

For instance, the sinusoidal terms usually encountered in many problems of robotics are all global Lipschitz. Moreover, most nonlinearities are local Lipschitz if they are considered in a given neighborhood (see the def. in [23]).

A2

$$
\begin{aligned}
& \left\{\begin{array}{c}
\operatorname{rank}\left[\begin{array}{ccc}
E_{\alpha(k+1)} & F_{\alpha(k)} & 0 \\
0 & G_{\alpha(k)} & 0 \\
C_{\alpha(k+1)} & 0 & G_{\alpha(k+1)}
\end{array}\right] \\
=n+\operatorname{rank} G_{\alpha(k+1)}+\operatorname{rank}\left[\begin{array}{l}
F_{\alpha(k)} \\
G_{\alpha(k)}
\end{array}\right]
\end{array}\right. \\
& \left\{\begin{array}{c}
\operatorname{rank}\left[\begin{array}{cc}
z E_{i}-A_{i} & -F_{i} \\
C_{i} & G_{i}
\end{array}\right]=n+\operatorname{rank}\left[\begin{array}{c}
F_{i} \\
G_{i}
\end{array}\right] \\
=\forall|z| \geq 1, \quad i \in \varepsilon
\end{array}\right. \\
& \left\{\begin{array}{c}
p+2 m>n+q+\operatorname{rank} G_{\alpha(k)}, \\
\operatorname{rank}\left[\begin{array}{l}
F_{\alpha(k)} \\
G_{\alpha(k)}
\end{array}\right]=q, \\
\operatorname{rank}\left[C_{\alpha(k)}\right.
\end{array} \quad G_{\alpha(k)}\right]=m
\end{aligned}
$$

Remark 2: Define $V_{1}=\left[\begin{array}{ccc}I_{n} & 0 & 0 \\ C_{\alpha(k+1)} & 0 & -I_{m} \\ 0 & I_{m} & 0\end{array}\right], V_{2}=$ $\left[\begin{array}{ccc}I_{n} & 0 & 0 \\ 0 & I_{q} & 0 \\ 0 & 0 & -I_{q}\end{array}\right]$ and $\Gamma=\left[\begin{array}{ccc}I_{n} & F_{\alpha(k)} & 0 \\ 0 & G_{\alpha(k)} & 0 \\ C_{\alpha(k+1)} & 0 & G_{\alpha(k+1)}\end{array}\right]$. For $E_{\alpha(k+1)} \stackrel{1}{=} I_{n}$, the assumption A2 becomes equivalent to assumption (12) given in [11] since

$$
\operatorname{rank} \Gamma=n+\operatorname{rank} G_{\alpha(k+1)}+\operatorname{rank}\left[\begin{array}{c}
F_{\alpha(k)} \\
G_{\alpha(k)}
\end{array}\right]
$$

is equivalent to

$$
\begin{gathered}
\operatorname{rank} V_{1} \Gamma V_{2}=\left[\begin{array}{ccc}
I_{n} & 0 & 0 \\
0 & I_{q} & 0 \\
0 & 0 & -I_{q}
\end{array}\right] \\
=n+\operatorname{rank} G_{\alpha(k+1)}+\operatorname{rank}\left[\begin{array}{l}
F_{\alpha(k)} \\
G_{\alpha(k)}
\end{array}\right]
\end{gathered}
$$

which is equivalent to

$$
\begin{aligned}
& \operatorname{rank}\left[\begin{array}{ccc}
I_{n} & F_{\alpha(k)} & 0 \\
0 & C_{\alpha(k+1)} F_{\alpha(k)} & G_{\alpha(k+1)} \\
0 & G_{\alpha(k)} & 0
\end{array}\right] \\
= & n+\operatorname{rank} G_{\alpha(k+1)}+\operatorname{rank}\left[\begin{array}{c}
F_{\alpha(k)} \\
G_{\alpha(k)}
\end{array}\right]
\end{aligned}
$$

which is equivalent to (12) given in [11]. In addition for $\alpha(k+1)=\alpha(k)$, the assumption $\mathrm{A} 2$ becomes equivalent to condition (1-1) given by [29].
Remark 3: The assumptions $\operatorname{rank}\left[\begin{array}{l}F_{\alpha(k)} \\ G_{\alpha(k)}\end{array}\right]=q$ and $\operatorname{rank}\left[\begin{array}{ll}C_{\alpha(k)} & G_{\alpha(k)}\end{array}\right]=m$ ensure respectively that the UI's and measurements are linearly independent. This can always be satisfied by redefining the UI and measurement vector [10]. While, according to remark $1, p+2 m>n+q+\operatorname{rank} G_{\alpha(k)}$ is necessary in order to ensure that $\Theta_{k_{+}}^{\perp}$ is well defined.

Our aim is to design an observer in the form,

$$
\begin{aligned}
& z_{k+1}=\Pi_{k_{+}} z_{k}+K_{k_{+}} y_{k}+T_{k_{+}} H_{\alpha(k)} \phi\left(\hat{x}_{k}, u_{k}, k\right) \\
& \hat{x}_{k}=z_{k}+N_{\alpha(k-1), \alpha(k)} y_{k}
\end{aligned}
$$

where $z_{k} \in \mathbb{R}^{n}$ and

$$
\left[\begin{array}{llll}
T_{k_{+}} & N_{k_{+}} & K_{1 k_{+}} & \Pi_{k_{+}}
\end{array}\right]=\Psi \Theta_{k_{+}}^{+}-Z_{\alpha(k)} \Theta_{k_{+}}^{\perp}
$$

with

$$
\begin{aligned}
& \Theta_{k_{+}}=\left[\begin{array}{cccc}
E_{\alpha(k+1)} & A_{\alpha(k)} & F_{\alpha(k)} & 0 \\
C_{\alpha(k+1)} & 0 & 0 & G_{\alpha(k+1)} \\
0 & -C_{\alpha(k)} & -G_{\alpha(k)} & 0 \\
0 & -I_{n} & 0 & 0
\end{array}\right] \\
& \Psi=\left[\begin{array}{ll}
I_{n} & 0_{n \times(n+2 q)}
\end{array}\right], \Theta_{k_{+}}^{\perp}=\left(I_{n+p+2 m}-\Theta_{k_{+}} \Theta_{k_{+}}^{+}\right) \\
& K_{k_{+}}=K_{1 k_{+}}+\Pi_{k_{+}} N_{\alpha(k-1), \alpha(k)}
\end{aligned}
$$

The problem of the observer design is also reduced to finding matrices $Z_{\alpha(k)}$ such that the estimate $\hat{x}_{k}$ converges asymptotically to the state $x_{k}$.

\section{OBSERVER DESIGN}

In this section, a new method is presented to design the observer (2) for switched nonlinear system (1). The following theorem will give the structure of the observer.

Theorem 1: Under A2, there exist matrices $T_{k_{+}}, N_{k_{+}}$, $K_{1 k_{+}}, \Pi_{k_{+}}$such that

$$
\begin{gathered}
T_{k_{+}} E_{\alpha(k+1)}+N_{k_{+}} C_{\alpha(k+1)}=I_{n} \\
\Pi_{k_{+}}=T_{k_{+}} A_{\alpha(k)}-K_{1 k_{+}} C_{\alpha(k)} \\
T_{k_{+}} F_{\alpha(k)}-K_{1 k_{+}} G_{\alpha(k)}=0 \\
N_{k_{+}} G_{\alpha(k+1)}=0
\end{gathered}
$$

and the difference of the state estimation error $e_{k}=x_{k}-\hat{x}_{k}$ becomes

$$
e_{k+1}=\Pi_{k_{+}} e_{k}+T_{k_{+}} H_{\alpha(k)} \tilde{\phi}_{k}
$$

where

$$
\begin{aligned}
\tilde{\phi}_{k} & =\phi\left(x_{k}, u_{k}, k\right)-\phi\left(\hat{x}_{k}, u_{k}, k\right) \\
K_{k_{+}} & =K_{1 k_{+}}+\Pi_{k_{+}} N_{\alpha(k-1), \alpha(k)}
\end{aligned}
$$

Remark 4: Consider the single system (1) where $\varepsilon=\{1\}$, $\alpha(k+1)=\alpha(k+1)=1, E_{\alpha(k+1)}=E_{1}, A_{\alpha(k)}=A_{1}$, $F_{\alpha(k)}=F_{1}, H_{\alpha(k)}=0, C_{\alpha(k)}=C_{1}$ and $G_{\alpha(k)}=G_{1}$. When $G_{1}$ has full row rank, the matrix $C_{12}$ defined in [10] is necessarily equal to zero. Consequently the matrices $N$ and $M$ defined by (24) and (25) in [10] cannot be computed and the observer is unfeasible. Furthermore in our approach, when $G_{1}$ has full row rank, it follow that the only $N_{1,1}$ which fulfills $N_{1,1} G_{1}=0$ is the zero matrix. So, the observer (2) is solvable provided the matrix $\Pi_{1,1}=T_{1,1} A_{1}-K_{1,1} C_{1}$ is stable, $T_{1,1} E_{1}=I_{n}$ and $K_{1_{1,1}} G_{1}=T_{1,1} F_{1}$. In other words, $E_{1}$ must be nonsingular $\left(T_{1,1}=E_{1}^{-1}\right)$ and the row image of 
$E_{1}^{-1} F_{1}$ has to be included in the row image of $G_{1}$ while the solution $K_{1,1}$ of $K_{1_{1,1}} G_{1}=E_{1}^{-1} F_{1}$ must ensure the stability of $\Pi_{1,1}=E_{1}^{-1} A_{1}-K_{1,1} C_{1}$. This is very restrictive, but a solution may exists. So, our observer may exist even if the number of UI in measurement equation is equal to the number of the measurement. In addition the detectability condition A3 is the usual condition defined in UIO theory, see for instance (23) in [10]. So the methodologies proposed is no less restrictive than those reported in the literature [8], [10], [11], [6], [29], [17].

Proof: Suppose that (3) hold, then $e_{k+1}=x_{k+1}-\hat{x}_{k+1}$ becomes

$$
e_{k+1}=T_{k_{+}} E_{\alpha(k+1)} x_{k+1}-z_{k+1}-N_{k_{+}} G_{\alpha(k+1)} d_{k+1}
$$

and from (1), (2) and (8), $e_{k+1}$ becomes

$$
\begin{aligned}
e_{k+1}= & \left(T_{k_{+}} A_{\alpha(k)}-\Pi_{k_{+}} T_{k_{+}} E_{\alpha(k)}-K_{k_{+}} C_{\alpha(k)}\right) x_{k} \\
& +\Pi_{k_{+}} e_{k}+T_{k_{+}} H_{\alpha(k)} \tilde{\phi}_{k}-N_{k_{+}} G_{\alpha(k+1)} d_{k+1} \\
& +\left(T_{k_{+}} F_{\alpha(k)}-\left(K_{k_{+}}-\Pi_{k_{+}} N_{\alpha(k-1), \alpha(k)}\right) G_{\alpha(k)}\right) d_{k}
\end{aligned}
$$

Substituting (9) into (10) and using the constraints (4-6), $T_{\alpha(k-1), \alpha(k)} E_{\alpha(k)}+N_{\alpha(k-1), \alpha(k)} C_{\alpha(k)}=I_{n}$ (7) is obtained. Rewriting (7) and $(3,4,5,6)$ respectively as

$$
\begin{aligned}
e_{k+1}= & {\left[\begin{array}{llll}
T_{k_{+}} & N_{k_{+}} & K_{1 k_{+}} & \Pi_{k_{+}}
\end{array}\right] \varphi_{1 \alpha(k)} e_{k} } \\
& +\left[\begin{array}{llll}
T_{k_{+}} & N_{k_{+}} & K_{1 k_{+}} & \Pi_{k_{+}}
\end{array} \varphi_{2 \alpha(k)} \tilde{\phi}_{k}\right. \\
\Psi= & {\left[\begin{array}{llll}
T_{k_{+}} & N_{k_{+}} & K_{1 k_{+}} & \Pi_{k_{+}}
\end{array}\right] \Theta_{k_{+}} }
\end{aligned}
$$

where

$$
\varphi_{1 \alpha(k)}=\left[\begin{array}{c}
A_{\alpha(k)} \\
0_{m \times n} \\
-C_{\alpha(k)} \\
0_{n \times n}
\end{array}\right], \varphi_{2 \alpha(k)}=\left[\begin{array}{c}
H_{\alpha(k)} \\
0_{m \times n} \\
0_{m \times n} \\
0_{n \times n}
\end{array}\right]
$$

The solution $\left[\begin{array}{llll}T_{k_{+}} & N_{k_{+}} & K_{1 k_{+}} & \Pi_{k_{+}}\end{array}\right]$of (12) depends on the rank of matrix $\Theta_{k_{+}}$. A solution exists if and only if [25]

$$
\operatorname{rank}\left[\begin{array}{c}
\Theta_{k_{+}} \\
\Psi
\end{array}\right]=\operatorname{rank} \Theta_{k_{+}}
$$

which is equivalent to $A 2$. Therefore, under $A 2$, the general solution of (12) is

$$
\left[\begin{array}{llll}
T_{k_{+}} & N_{k_{+}} & K_{1 k_{+}} & \Pi_{k_{+}}
\end{array}\right]=\Psi \Theta_{k_{+}}^{+}-Z_{\alpha(k)} \Theta_{k_{+}}^{\perp}
$$

where $\Theta_{k_{+}}^{\perp}=\left(I_{n+p+2 m}-\Theta_{k_{+}} \Theta_{k_{+}}^{+}\right)$and $Z_{\alpha(k)}$ is an arbitrary matrix of appropriate dimension.

Substituting (14) into (11) gives (7), where $\Pi_{k_{+}}$and $T_{k_{+}}$ are determined by known matrices and by the arbitrary matrix $Z_{\alpha(k)}$ as follows

$$
\begin{aligned}
\Pi_{k_{+}} & =\Psi \Theta_{k_{+}}^{+} \varphi_{1 \alpha(k)}-Z_{\alpha(k)} \Theta_{k_{+}}^{\perp} \varphi_{1 \alpha(k)} \\
T_{k_{+}} H_{\alpha(k)} & =\Psi \Theta_{k_{+}}^{+} \varphi_{2 \alpha(k)}-Z_{\alpha(k)} \Theta_{k_{+}}^{\perp} \varphi_{2 \alpha(k)}
\end{aligned}
$$

Now, the condition of global stability of (7) is stated in the following theorem.

Theorem 2: If there exist symmetric positive definite ma- trices $P_{1}, P_{2}, . . P_{h}$ and matrices $U_{1}, U_{2}, . . U_{h}$ satisfying

$$
\left[\begin{array}{cccc}
P_{i}+P_{i}^{T}-P_{j} & X_{1} & X_{2} & 0 \\
* & P_{i} & 0 & \gamma I_{n} \\
* & * & I_{n} & 0 \\
* & * & * & I_{n}
\end{array}\right]>0, \forall i, j \in \varepsilon
$$

then the state estimation error $e_{k}$ converges globally towards the origins. $X_{1,2}=P_{i} \Psi \Theta_{i, j}^{+} \varphi_{1 i}-U_{i} \Theta_{i, j}^{\perp} \varphi_{1 i}$ and $X_{2}=$ $P_{i} \Psi \Theta_{i, j}^{+} \varphi_{2 i}-U_{i} \Theta_{i, j}^{\perp} \varphi_{2 i}$. Moreover, the resulting observer gains are given by (14) and (9), where the matrices $Z_{i}$ are given by $Z_{i}=P_{i}^{-1} U_{i}$.

Proof: Consider the switched Lyapunov function $V\left(e_{k}, k\right)=e_{k}^{T} P_{\alpha(k)} e_{k}$ where $P_{\alpha(k)}>0$ is a positive definite matrix. If such a Lyapunov function exists and its difference $\Delta V=V\left(e_{k+1}, k+1\right)-V\left(e_{k}, k\right)$ is negative definite along system trajectories of (7), then the origin of the system (7) is globally asymptotically stable. Computing the difference $\Delta V$, along the solution of (7), $\Delta V$ is given by

$$
\begin{aligned}
& \Delta V=e_{k+1}^{T} P_{\alpha(k+1)} e_{k+1}-e_{k}^{T} P_{\alpha(k)} e_{k} \\
&=e_{k}^{T} \Pi_{k_{+}}^{T} P_{\alpha(k+1)} \Pi_{k_{+}} e_{k}-e_{k}^{T} P_{\alpha(k)} e_{k} \\
&+2 e_{k}^{T} \Pi_{k_{+}}^{T} P_{\alpha(k+1)} T_{k_{+}} H_{\alpha(k)} \tilde{\phi}_{k} \\
&+\tilde{\phi}_{k}^{T} H_{\alpha(k)}^{T} T_{k_{+}}^{T} P_{\alpha(k+1)} T_{k_{+}} H_{\alpha(k)} \tilde{\phi}_{k} \\
& \leq e_{k}^{T} \Pi_{k_{+}}^{T} P_{\alpha(k+1)} \Pi_{k_{+}} e_{k} \\
&+2 e_{k}^{T} \Pi_{k_{+}}^{T} P_{\alpha(k+1)} T_{k_{+}} H_{\alpha(k)} \tilde{\phi}_{k} \\
&+\tilde{\phi}_{k}^{T} H_{\alpha(k)}^{T} T_{k_{+}}^{T} P_{\alpha(k+1)} T_{k_{+}} H_{\alpha(k)} \tilde{\phi}(k) \\
&-e_{k}^{T} P_{\alpha(k)} e_{k}-\tilde{\phi}_{k}^{T} \tilde{\phi}_{k}+\gamma^{2} e_{k}^{T} e_{k}
\end{aligned}
$$

since from A1 and (8) we have $-\tilde{\phi}_{k}^{T} \tilde{\phi}_{k}+\gamma^{2} e_{k}^{T} e_{k} \geq 0$.

Now, $\Delta V$ can be written as

$\Delta V\left(e_{k}, k\right) \leq e_{a_{k}}^{T}\left[\begin{array}{cc}\Gamma_{k_{+}} & \Pi_{k_{+}}^{T} P_{\alpha(k+1)} T_{k_{+}} H_{\alpha(k)} \\ * & H_{\alpha(k)}^{T} T_{k_{+}}^{T^{+}} P_{\alpha(k+1)} T_{k_{+}} H_{\alpha(k)}-I_{n_{\phi}}\end{array}\right] e_{a_{k}}$ where $\Gamma_{k_{+}}=\Pi_{k_{+}}^{T} P_{\alpha(k+1)} \Pi_{k_{+}}-P_{\alpha(k)}+\gamma^{2} I_{n}$ and $e_{a_{k}}^{T}=$ $\left[\begin{array}{cc}e_{k}^{T} & \tilde{\phi}_{k}^{T}\end{array}\right]$. The difference $\Delta V\left(e_{k}, k\right)$ is negative definite for any $\left[\begin{array}{ll}e_{k}^{T} & \tilde{\phi}_{k}^{T}\end{array}\right] \neq 0$ if

$$
\left[\begin{array}{cc}
\Gamma_{k_{+}} & \Pi_{k_{+}}^{T} P_{\alpha(k+1)} T_{k_{+}} H_{\alpha(k)} \\
* & H_{\alpha(k)}^{T} T_{k_{+}}^{T} P_{\alpha(k+1)} T_{k_{+}} H_{\alpha(k)}-I_{n_{\phi}}
\end{array}\right]<0
$$

As this inequality has to be satisfied under arbitrary switching law, it follows that it should hold for special configuration $\alpha(k+1)=j$ and $\alpha(k)=i$. Define $X_{3}=P_{i}-\Pi_{i, j}^{T} P_{j} \Pi_{i, j}-$ $\gamma^{2} I_{n}$, then (18), becomes

$$
\left[\begin{array}{cc}
X_{3} & -\Pi_{i, j}^{T} P_{j} T_{i, j} H_{i} \\
* & -H_{i}^{T} T_{i, j}^{T} P_{j} T_{i, j} H_{i}+I_{n_{\phi}}
\end{array}\right]>0 \quad \forall i, j \in \varepsilon
$$

which is equivalent, by Schur complement, to

$$
\left[\begin{array}{ccc}
P_{j} & P_{j} \Pi_{i, j} & P_{j} T_{i, j} H_{i} \\
* & P_{i}-\gamma^{2} I_{n} & 0 \\
* & * & I_{n_{\phi}}
\end{array}\right]>0 \quad \forall i, j \in \varepsilon
$$

which is equivalent, by Schur complement, to

$$
\left[\begin{array}{cccc}
P_{j} & P_{j} \Pi_{i, j} & P_{j} T_{i, j} H_{i} & 0 \\
* & P_{i} & 0 & \gamma I_{n} \\
* & * & I_{n_{\phi}} & 0 \\
* & * & * & I_{n}
\end{array}\right]>0 \quad \forall i, j \in \varepsilon
$$


which is equivalent by Lemma 1 of [31] to

$$
\left[\begin{array}{cccc}
P_{i}+P_{i}^{T}-P_{j} & P_{i} \Pi_{i, j} & P_{i} T_{i, j} H_{i} & 0 \\
\hline * & P_{i} & 0 & \gamma I_{n} \\
* & * & I_{n_{\phi}} & 0 \\
* & * & * & I_{n}
\end{array}\right]>0(20)
$$

where the matrices $S, M, Q$ and $G$ in [31] are directly identified by $S=P_{j}, M^{T}=\left[\begin{array}{lll}\Pi_{i, j} & T_{i, j} H_{i} & 0\end{array}\right], Q=$ $\left[\begin{array}{ccc}P_{i} & 0 & \gamma I_{n} \\ * & I_{n_{\phi}} & 0 \\ * & * & I_{n}\end{array}\right]$ and $G=P_{i}$. Substituting (16) and $U_{i}=$ $\bar{P}_{i} Z_{i}$ into (20) with $\alpha(k)=i$ and $\alpha(k+1)=j$, (17) is obtained.

Remark 5: The feasibility of (17), or equivalently of (19), implies that the pairs $\left(\Psi \Theta_{i, i}^{+} \varphi_{1 i}, \Theta_{i, i}^{\perp} \varphi_{1 i}\right)$ are detectable. Indeed, according to Theorem 2, satisfying (17) is equivalent to guarantee the stability of the state estimation error (7) whatever the switching rule can be. This includes the case where the switching rule leads to a linear behavior, i.e. $\alpha(k+1)=\alpha(k)=i$ and $\Pi_{i, i}$ has to be Hurwitz. In other words for $\alpha(k+1)=\alpha(k)=i$, the existence of a solution $P_{i}>0, U_{i}$, of the LMI (17) needs that the matrix $\Pi_{i, i}=\Psi \Theta_{i, i}^{+} \varphi_{1 i}-Z_{i} \Theta_{i, i}^{\perp} \varphi_{1 i}$ is Hurwitz (in the meaning of Lyapunov stability) since the element $(1,1)$ of (19) implies $-P_{i}+\Pi_{i, i}^{T} P_{i} \Pi_{i, i}<-\gamma^{2} I_{n+m}<0$.

Remark 6: Of course, the switched detectability of the system (7) is not ensured by the assumption that for each subsystem $i \in \varepsilon$, the pair $\left(\Psi \Theta_{i, i}^{+} \varphi_{1 i}, \Theta_{i, i}^{\perp} \varphi_{1 i}\right)$ is detectable (see the example in section 7.2 given by ([4]), but the detectability of each pair $\left(\Psi \Theta_{i, i}^{+} \varphi_{1 i}, \Theta_{i, i}^{\perp} \varphi_{1 i}\right)$ is a necessary condition to solve (17). Moreover an arbitrary choice of $T_{i, i}$ can involve a loss of detectability of the pair $\left(\Psi \Theta_{i, i}^{+} \varphi_{1 i}, \Theta_{i, i}^{\perp} \varphi_{1 i}\right)$ (see [9]). To overcome the problem of an arbitrary choice of $T_{i, i}$ the computation of a suitable $T_{i, i}$ is included in the design procedure. That is why (7) is rewritten as (11) where $\left[\begin{array}{llll}T_{k_{+}} & N_{k_{+}} & K_{1 k_{+}} & \Pi_{k_{+}}\end{array}\right]$is given by (14). Thus the matrix $Z_{i}$ involved in $T_{i, i}$ (14) plays the role of a parametrization. The switched observer design is finally reduced to the computation of the gain matrices $Z_{i}, i \in \varepsilon$, ensuring the asymptotic stability of system (7) under arbitrary switching signal.

Now the following results can be established.

Lemma 1: There exist matrices $Z_{i}$ such that the matrices $\Pi_{i, i}=\Psi \Theta_{i, i}^{+} \varphi_{1 i}-Z_{i} \Theta_{i, i}^{\perp} \varphi_{1 i}$ is Hurwitz if and only if the pair $\left(\Psi \Theta_{i, i}^{+} \varphi_{1 i}, \Theta_{i, i}^{\perp} \varphi_{1 i}\right)$ is detectable, which is equivalent to (21), which is equivalent to A3.

$$
\operatorname{rank}\left[\begin{array}{c}
z I_{n}-\Psi \Theta_{i, i}^{+} \varphi_{1 i} \\
\Theta_{i, i}^{\perp} \varphi_{1 i}
\end{array}\right]=n, \forall|z| \geq 1
$$

Proof: See the appendix.

\section{EXAMPLES}

In this section, the results are illustrated with two simulations. In the first one, the studied system is nonsingular, it is derived from the continuous system of [24], while the second simulation concerns a switched systems subject to UI, nonlinearities and algebraic constraints.
Example 1: From [24] the observer (2) for system (1a,1b) is guaranteed to be stable for all nonlinearities with Lipschitz constant of magnitude less than 0.49. Using our above LMI formulation, it is proposed to find the largest Lipschitz constant $\gamma$ such that the observer (2) exists for system (1). The system $(1 \mathrm{a}, 1 \mathrm{~b})$ considered in [24] is in first approximated by the Euler approximation where for a good approximation the sample time is fixed to $T_{e}=0.01 \mathrm{~s}$. Let consider the discrete-time model (1) where $\varepsilon=\{1\}, \alpha(k)=1 \forall k, E_{1}=I, A_{1}=$ $I_{2}+T_{e} \bar{A}, \bar{A}=\left[\begin{array}{cc}0 & 1 \\ 1 & -1\end{array}\right], F_{1}=0, G_{1}=0, H_{1}=T_{e} I_{2}$ and $C_{1}=\left[\begin{array}{ll}0 & 1\end{array}\right]$. It is assumed that the nonlinearity $\phi\left(x_{k}, u_{k}, k\right)$ is globally Lipschitz in $x_{k}$ with Lipschitz constant $\gamma$ and $A 2$ holds since $E=I_{2}$ and $A 3$ holds for all $z$.

For a known Lipschitz constant $\gamma$, the Theorem 2 gives the gain observer $Z_{1}$ such that observer (2) for system (1) exists. Theorem 2 can be reformulated as the following convex optimization problem

$$
\max _{P_{1}, U_{1}} \gamma \text { subject to }(17) \text { and } P_{1}=P_{1}^{T}>0
$$

where $i=j=1, \Theta_{k_{+}}=\left[\begin{array}{cc}I_{2} & A_{1} \\ C_{1} & 0 \\ 0 & -C_{1} \\ 0 & -I_{2}\end{array}\right]$ and $\Psi=\left[\begin{array}{ll}I_{n} & 0_{n \times n}\end{array}\right]$. Applying the convex optimization problem defined by (22), the following results are obtained: $\gamma=0.9950, T_{1,1}=$ $\left[\begin{array}{ll}1 & -9.99 \\ 0 & 0.0141\end{array}\right], N_{1,1}=\left[\begin{array}{c}9.99 \\ 0.9859\end{array}\right], K_{1,1}=\left[\begin{array}{c}-0.8881 \\ 0.0152\end{array}\right]$ and $\Pi_{1,1}=\left[\begin{array}{cc}0.9001 & 0 \\ 0.0001 & 0.0047\end{array}\right]$. It can be noted that the maximal constant of Lipschitz obtained by the present approach is larger than the Lipschitz constant given by [24]. If $C_{1}=\left[\begin{array}{ll}1 & 0\end{array}\right]$, the maximal constant of Lipschitz is $\gamma=1.4142$.

The following example shows that a switched observer may exist for a more general class.

Example 2: Consider the switched nonlinear descriptor systems (1) where

$$
\begin{gathered}
E_{i}=\left[\begin{array}{llll}
1 & 0 & 0 & 0 \\
0 & 1 & 0 & 0 \\
0 & 0 & 1 & 0 \\
0 & 0 & 0 & 0
\end{array}\right], F_{i}=\left[\begin{array}{ll}
f_{11_{i}} & 0 \\
0 & f_{22_{i}} \\
0 & 0 \\
0 & 1
\end{array}\right], \\
A_{i}=\left[\begin{array}{llll}
-1 & a_{12_{i}} & 0 & a_{14_{i}} \\
-1 & 0 & 0 & 1 \\
0 & -1 & a_{33_{i}} & 0 \\
0 & 0 & 0 & 0.5
\end{array}\right], x_{k}=\left[\begin{array}{l}
x_{1 k} \\
x_{2 k} \\
x_{3 k} \\
x_{4 k}
\end{array}\right]
\end{gathered}
$$$$
H_{i}=\left[\begin{array}{l}
1 \\
0 \\
h_{31_{i}} \\
0
\end{array}\right], \phi_{k}=\gamma \sin x_{1 k}, C_{i}=\left[\begin{array}{llll}
1 & 0 & 0 & 0 \\
0 & 0 & c_{23_{i}} & 1 \\
0 & 0 & 0 & c_{34_{i}}
\end{array}\right]
$$

$$
\begin{gathered}
G_{i}=\left[\begin{array}{cc}
1 & 0 \\
0 & 0 \\
1 & 0
\end{array}\right], d_{k}=\left[\begin{array}{c}
d_{1 k} \\
d_{2 k}
\end{array}\right], \varepsilon=\{1,2\}, \gamma=0.5, \\
T_{e}=0.01 \mathrm{sec}, d_{1 k}=\sin 4 k T_{e}, d_{2 k}=\sin 0.1 k T_{e}, \\
a_{12_{1}}=0.4, a_{12_{2}}=0.6, a_{33_{1}}=-0.4, a_{33_{2}}=-0.6,
\end{gathered}
$$




$$
\begin{gathered}
a_{14_{1}}=0.2, a_{14_{2}}=0, c_{23_{1}}=1, c_{23_{2}}=0, c_{34_{1}}=1, c_{34_{2}}=0, \\
h_{31_{1}}=1, h_{31_{2}}=0, f_{11_{1}}=0, f_{11_{2}}=1, f_{22_{1}}=1, f_{22_{2}}=0
\end{gathered}
$$

and where the switching time sequence is given by table 1 .

\begin{tabular}{l|l|l|l|l|l|l|l|l|l|l|l|l}
$k$ & 0 & $\ldots$ & 49 & 50 & 51 & 52 & $\ldots$ & 250 & 251 & 252 & 253 & $\ldots$ \\
\hline$\alpha(k-1)$ & 2 & $\ldots$ & 2 & 2 & 2 & 1 & $\ldots$ & 1 & 1 & 1 & 2 & $\ldots$ \\
\hline$\alpha(k)$ & 2 & $\ldots$ & 2 & 2 & 1 & 1 & $\ldots$ & 1 & 1 & 2 & 2 & $\ldots$ \\
\hline$\alpha(k+1)$ & 2 & $\ldots$ & 2 & 1 & 1 & 1 & $\ldots$ & 1 & 2 & 2 & 2 & $\ldots$
\end{tabular}

TABLE I

SWITCHING SEQUENCE

Remark 7: If the switching time sequence is unknown a priori, a switching rule can be defined, for instance see example 1 in [20].

\section{Algorithm}

1) The assumption $A 1$ holds for $\gamma=0.5$. Assumption $A 2$ holds for all couples $\{(2,2) ;(2,1) ;(1,1) ;(2,2)\}$, for instance $\alpha(k+1)=2, \alpha(k)=1$, and the equality

$$
\left[\begin{array}{ccc}
E_{2} & F_{1} & 0 \\
0 & G_{1} & 0 \\
C_{2} & 0 & G_{2}
\end{array}\right]=n+\operatorname{rank} G_{2}+\operatorname{rank}\left[\begin{array}{c}
F_{1} \\
G_{1}
\end{array}\right]
$$

is satisfied. The assumption $A 3$ holds for all $|z| \geq 1$ and for all $i \in \varepsilon=\{1,2\}$.

2) From table $1, \varphi_{1_{1}}, \varphi_{2_{1}}, \varphi_{1_{2}}, \varphi_{2_{2}}, \Theta_{2,2}, \Theta_{2,1}, \Theta_{1,1}, \Theta_{1,2}$ and $\Psi$ are computed. Since assumptions A1, A2, A3 and A4 hold, one can solve the convex optimization problem defined in theorem 2 . More precisely, finding $P_{1}, P_{2}, U_{1}, U_{2}$ subject to $P_{1}=P_{1}^{T}>0, P_{2}=P_{2}^{T}>0$, (17) with $i, j=2,2$, (17) with $i, j=2,1$, (17) with $i, j=1,1$ and (17) with $i, j=1,2$. After 26 iterations, the gains $Z_{1}, Z_{2}$ are obtained. From (14) we deduce

$$
\begin{aligned}
& {\left[\begin{array}{llll}
T_{2,2} & N_{2,2} & K_{1_{2,2}} & \Pi_{2,2}
\end{array}\right]=\Psi \Theta_{2,2}^{+}-Z_{2} \Theta_{2,2}^{\perp}} \\
& {\left[\begin{array}{llll}
T_{2,1} & N_{2,1} & K_{1_{2,1}} & \Pi_{2,1}
\end{array}\right]=\Psi \Theta_{2,1}^{+}-Z_{2} \Theta_{2,1}^{\perp}} \\
& {\left[\begin{array}{llll}
T_{1,1} & N_{1,1} & K_{1_{1,1}} & \Pi_{1,1}
\end{array}\right]=\Psi \Theta_{1,1}^{+}-Z_{1} \Theta_{1,1}^{\perp}} \\
& {\left[\begin{array}{llll}
T_{1,2} & N_{1,2} & K_{1_{1,2}} & \Pi_{1,2}
\end{array}\right]=\Psi \Theta_{1,2}^{+}-Z_{1} \Theta_{1,2}^{\perp}}
\end{aligned}
$$

3) Using the matlab/simulink software two S-functions are written, the first for system (1) and second for the observer (2). According to table 1 , the matrices $T_{k_{+}}$, $K_{k_{+}}, \Pi_{k_{+}}$and $N_{\alpha(k-1), \alpha(k)}$ are updated with $K_{k_{+}}=$ $K_{1 k_{+}}+\Pi_{k_{+}} N_{\alpha(k-1), \alpha(k)}$.

Simulation results show, through figures 1(a), 1(b) a good state estimation performance. The estimation of the state $x_{4}$ is not presented due to space limitation.

Remark 8: If a common quadratic Lyapunov function $V\left(e_{k}, k\right)=e_{k}^{T} P e_{k}$ is imposed (i.e., $P_{1}=P_{2}=P$ and $U_{1}=$ $U_{2}=U$, , the corresponding LMI are found to be unfeasible. Indeed, the polyquadratic stability is less conservative than the quadratic stability.

Remark 9: If the convex optimization, defined by (22), is applied, the following maximal bound $\gamma$ is obtained for different value of $h_{31_{1}}$ :

\begin{tabular}{l|l|l|l|l|l}
$h_{31_{1}}$ & 1 & 1.2 & 1.26 & 1.27 & 1.28 \\
\hline$\gamma_{\max }$ & 184.3 & 98.87 & 49.53 & 35.05 & 1.1768
\end{tabular}
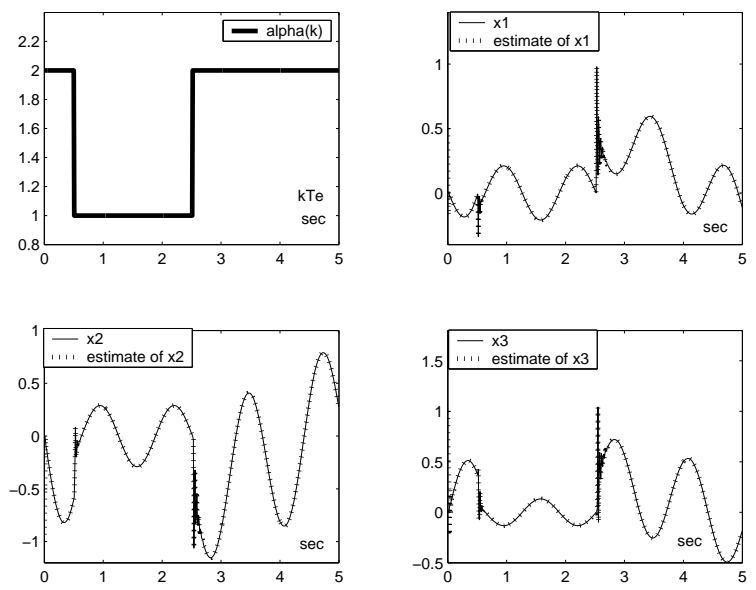

(a) Switching time sequence and state estimation
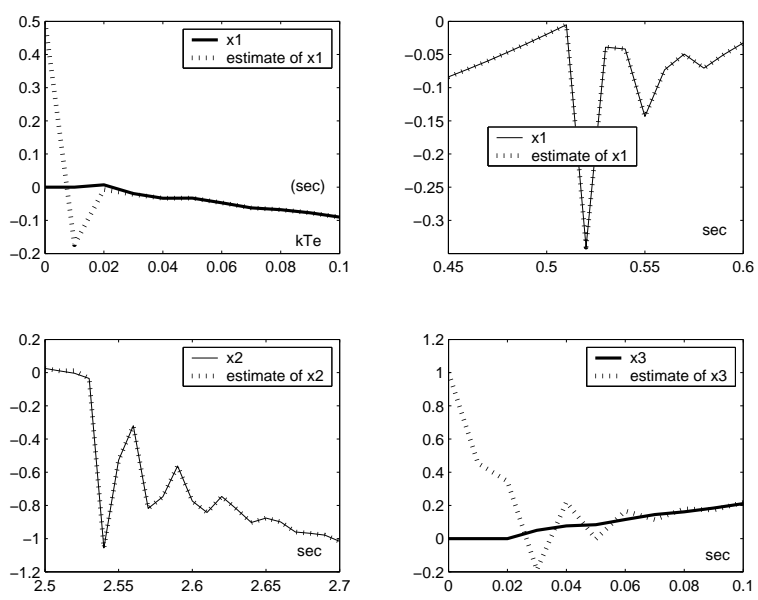

(b) zoom of the state estimation

Fig. 1. Switching time sequence and state estimation performance

where the parameter $h_{31_{1}}$ is a coefficient of the matrix $H_{\alpha(k)}$ of system (1). If $h_{31_{1}}$ increases then $\gamma_{\max }$ decreases since there is linear dependant of the nonlinear term $\phi\left(x_{k}, u_{k}, k\right)$.

\section{CONCLUSION}

A rigorous method for the design of observers for switched nonlinear descriptor systems in the presence of UI has been presented. Existence conditions of such observers have been given and proved with a strict LMI formulation. Furthermore a polyquadratic stability is used to assess state estimation. It is interesting to note that the systems addressed in this paper are of a more general class than those reported in the literature. Moreover, from [27] an extension to design a robust observer for an uncertain switched descriptor system can be developed, this is actually studied.

\section{APPENDIX}

It is proved that assumption A3, or (21) are equivalent to the existence of matrices $Z_{i}$ such that $\Pi_{i, i}$ are Hurwitz.

Proof $A 3 \Leftrightarrow(21)$. Define the following nonsingular matrices $W_{1 i}, W_{3}$ and the full-column rank matrix $W_{2 i}$

$W_{1 i}=\left[\begin{array}{ll}I_{n} & 0 \\ -\Theta_{i, i}^{+} \varphi_{1 i} & I_{2(n+q)}\end{array}\right], W_{2 i}=\left[\begin{array}{ll}I_{n} & -\Psi \Theta_{i, i}^{+} \\ 0 & \Theta_{i, i}^{\perp} \\ 0 & \Theta_{i, i} \Theta_{i, i}^{+}\end{array}\right]$, 


$$
W_{3}=\left[\begin{array}{ccccc}
-I_{n} & 0 & 0 & 0 & 0 \\
z I_{n} & I_{n} & 0 & 0 & 0 \\
0 & 0 & I_{n} & 0 & 0 \\
0 & 0 & 0 & -I_{q} & 0 \\
0 & 0 & 0 & z I_{q} & I_{q}
\end{array}\right]
$$

According to remark 5, for $\alpha(k+1)=\alpha(k)=i$ the existence of a solution $P_{i}>0, U_{i}$, of the LMI (17) needs that the matrix $\Pi_{i, i}$ is Hurwitz, therefore each pair $\left(\Psi \Theta_{i, i}^{+} \varphi_{1 i}, \quad \Theta_{i, i}^{\perp} \varphi_{1 i}\right)$ must be detectable. The proof is decomposed in two parts.

1) Let prove that $\mathrm{A} 3$ is equivalent to

$$
\begin{aligned}
& \operatorname{rank}\left[\begin{array}{ll}
z I_{n} & \Psi \\
\varphi_{1 i} & \Theta_{i, i}
\end{array}\right]-2 n-\operatorname{rank} G_{i} \\
& =n+q, \forall|z| \geq 1, \quad i \in \varepsilon
\end{aligned}
$$

2) Let prove that (23) is equivalent to (21).

Proof 1) From $W_{3}$, the relation (23) is equivalent to

$$
\begin{aligned}
& \operatorname{rank}\left[\begin{array}{ll}
z I_{n} & \Psi \\
\varphi_{1 i} & \Theta_{i}
\end{array}\right] W_{3}-2 n-\operatorname{rank} G_{i}=n+q, \\
& \forall|z| \geq 1, \quad i \in \varepsilon
\end{aligned}
$$

which is equivalent to

$$
\begin{aligned}
& \operatorname{rank}\left[\begin{array}{ccc}
z E_{i}-A_{i} & -F_{i} & 0 \\
z C_{i} & z G_{i} & G_{i} \\
C_{i} & G_{i} & 0
\end{array}\right]-\operatorname{rank} G_{i} \\
& =n+q, \forall|z| \geq 1, \quad i \in \varepsilon
\end{aligned}
$$

which is equivalent to $A 3$.

Proof 2) Since $\Theta_{i, i}^{+} \Theta_{i, i} \Theta_{i, i}^{+}=\Theta_{i, i}^{+}, \Theta_{i, i} \Theta_{i, i}^{+} \Theta_{i, i}=\Theta_{i, i}$ and $\operatorname{rank}\left[\begin{array}{c}\Theta_{i, i} \\ \Psi\end{array}\right]=\operatorname{rank} \Theta_{i, i}$, we obtain $(23)$

$$
\begin{array}{ll}
\Leftrightarrow \quad \operatorname{rank} W_{2 i}\left[\begin{array}{ll}
z I_{n} & \Psi \\
\varphi_{1 i} & \Theta_{i, i}
\end{array}\right] W_{1 i}-2 n-\operatorname{rank} G_{i} \\
=\quad n+q, \forall|z| \geq 1, \quad i \in \varepsilon \\
\Leftrightarrow \quad \operatorname{rank} \Theta_{i, i}+\operatorname{rank}\left[\begin{array}{l}
z I_{n+m}-\Psi \Theta_{i, i}^{+} \varphi_{1 i} \\
\Theta_{i, i}^{\perp} \varphi_{1 i}
\end{array}\right] \\
\quad \quad-2 n-\operatorname{rank} G_{i} \\
=\quad n+q, \forall|z| \geq 1, \quad i \in \varepsilon \\
\Leftrightarrow \quad \operatorname{rank}\left[\begin{array}{c}
F_{i} \\
G_{i}
\end{array}\right]+\operatorname{rank}\left[\begin{array}{l}
z I_{n+m}-\Psi \Theta_{i, i}^{+} \varphi_{1 i} \\
\Theta_{i, i}^{\perp} \varphi_{1 i}
\end{array}\right] \\
=\quad n+q, \forall|z| \geq 1, \quad i \in \varepsilon \\
\Leftrightarrow \quad(21)
\end{array}
$$

where $\operatorname{rank} \Theta_{i, i}=2 n+\operatorname{rank} G_{i}+\operatorname{rank}\left[\begin{array}{c}F_{i} \\ G_{i}\end{array}\right]$ and $\operatorname{rank}\left[\begin{array}{c}F_{i} \\ G_{i}\end{array}\right]=q$.

\section{REFERENCES}

[1] C. Aboki, G. Sallet and J.-C. Vivalda, "Obsevers for Lipschitz nonlinear systems," Int. J. Contr., vol. 75, no. 3, pp. 204-212, 2002.

[2] J. Agrawal, K. M. Moudgalya and A. K. Pani, "Sliding motion of discontinuous dynamical systems described by differential algebraic equations," IFAC Safeprocess, pp. 795-800, Washington, USA, 2003.

[3] G. Besançon and H. Hammouri, "On uniform observation of nonuniformaly observable systems," Sys. Contr. Lett. vol. 29, pp. 9-19, 1996.

[4] G. Böker and J. Lunze, "Stability and performance of swiching Kalman filters," Int. J. Contr., vol. 75, no. 16/17, pp. 1269-1281, 2002.

[5] Y. M. Cho and R. Rajamani, "A systematic approach to adaptive observer synthesis for NL systems," IEEE Trans. Automat. Contr., vol. 42, no. 4, pp. 534-537, 1997.
[6] D. Chu and V. Mehrmann, "Dirturbance decoupled observer design for descriptor systems," Sys. Contr. Lett. vol. 38, pp. 37-48, 1999.

[7] J. Daafouz, P. Riedinger and C. Iung, "Stability Analysis and Control Synthesis for Switched Systems: A Switched Lyapunov Function Approach," IEEE Trans. Automat. Contr, vol. AC-47, no. 11, pp. 18831887, Nov. 2002.

[8] M. Darouach, M. Zasadzinski, and D. Mehdi, "State estimation of stochastic singular linear systems," Int. J. Syst. Sci., vol. 2, no. 2, pp. 345-354, 1993.

[9] M. Darouach, M. Zasadzinski and S. J. Xu, "Full-order observers for linear systems with unknown inputs," IEEE Trans. on Automat. Contr., vol. 39, no. 3, pp. 606-609, 1994.

[10] M. Darouach, M. Zasadzinski and M. Hayar, "Reduced-order observer design for descriptor systems with unknown inputs," IEEE Trans. Automat. Contr., vol. 41, no. 7, pp. 1068-1072, 1996.

[11] M. Darouach, M. Zasadzinski and M. Boutayeb, "Extension of minimum variance estimation for systems with unknown inputs," Automatica, vol. 39, pp. 867-876, 2003.

[12] D. M. Dawson, "On the state observation and output feedback problems for nonlinear uncertain dynamic systems," Syst. Control Lett., vol. 18, pp. 217-222, 1992.

[13] A. J. Kerner and A. Isodori, "Linearization by output injection and nonlinear observers," Syst. Control Lett., vol. 3, pp. 47-52, 1983.

[14] A. J. Kerner and W. Respondek, "Nonlinear observers with linearizable error dynamics," SIAM Journal on Control and Optimization, vol. 23, no. 2, pp. 197-216, 1985.

[15] H. K. Khalil and F. Esfandiari, "Semiglobal stabilization of a class of nonlinear systems using output feedback," IEEE Trans. on Automat. Contr., vol. 38, pp. 1412-1415, 1993.

[16] D. Koenig, ”Unknown input proportional multiple-integral observer design for linear descriptor systems: application to state and fault estimation," IEEE Transaction of Automatic and Control, Vol. 50, no. 2, pp. $212-217$, Feb. 2005.

[17] D. Koenig, "Observer design for unknown input nonlinear descriptor systems via convex optimization," IEEE Transaction of Automatic and Control, vol. 51, no. 6, pp. 1047 - 1052, June 2006.

[18] D. Lieberzon and A. S. Morse, "Basic problems in stability and design of switching system," IEEE Control Syst. Mag., vol. 19, no. 5, pp. 59-70, oct. 1999.

[19] R. Marino, "Adaptive observers for single-output nonlinear systems," IEEE Trans. on Automat. Contr., vol. 35, pp. 1054-1058, 1990.

[20] G. Millerioux and J. Daafouz, "Unknown input observers for switched linear discrete time systems," Proceeding of the American Control Conference, pp. 5802-5805, Massachussets, USA, June 2004.

[21] R. J. Patton, R. N. Clark and P. M. Frank, "Fault diagnosis in dynamic systems," Englewood Cliffs, NJ: Prentice-Hall.

[22] L. Praly and Z. P. Jiang, "Stabilization by output feedback for systems with ISS inverse dynamics," Syst. Control Lett., vol. 21, pp. 19-33, 1993.

[23] S. Raghavan and J. K. Hedrick, "Observer design for a class of nonlinear systems," Int. J. Control, vol. 59, no. 2, pp. 515-528, 1994.

[24] R. Rajamani, "Observers for Lipschitz Nonlinear Systems," IEEE Trans. on Automat. Contr., vol. 43, no. 3, pp. 397-401, 1998.

[25] C. R. Rao and S.K. Mitra, "Generalized Inverse of Matrices and Its applications," New York: Wiley, 1971.

[26] Z. Sun and S.S. Ge, "Analysis and synthesis of switched linear control systems," Automatica, vol. 41, pp. 181-195, 2005

[27] Y. G. Sun, L. Wang and G. Xie, "Delay-dependent robust stability and stabilization for discrete-time switched with mode-dependent timevarying delays," Applied mathematics and computation, Elsevier, Article in press, 2006.

[28] A. Teel and L. Praly, "Global stabilizability and observability imply semi-global stabilizability by output feedback," Sys. Contr. Lett. vol. 22, pp. 313-325, 1994.

[29] M. E. Valcher, "State observers for discrete-time linear systems with unknown inputs," IEEE Trans. on Automat. Contr., vol. 44, no. 2, pp. 397-401, 1999.

[30] X. H. Xiao and W. Gao, "Nonlinear observer design by observer error linearization", SIAM Journal on Control and Optimization, vol. 27, no. 1, pp. 199-216, 1989.

[31] G. Xie and L. Wang, "Quadratic stability and stabilization of discretetime systems with state delay," Proceeding of the Conf. on Dec. and Contr., pp. 3235-3240, Bahamas, USA, Dec. 2004.

[32] F., Zhu and Z. Han, "A note on observers for Lipschitz nonlinear systems," IEEE Trans. on Automat. Contr., vol. 47, no. 10, pp. 17511754, 2002. 